\title{
The Prevalence of Vitamin D Deficiency in Patients with Myocardial Infarction
}

\author{
Salman $\mathbf{M}^{1^{*}}$, Al-Khoury $\mathrm{J}^{2}$, Al-Sherooqi $\mathbf{R}^{2}$ and Assar $\mathbf{W}^{3}$ \\ ${ }^{1}$ Northern Ireland Medical and Dental Training Agency (NIMDTA), Belfast, Ireland, UK \\ ${ }^{2}$ Department of Internal Medicine, King Hamad University Hospital, Kingdom of Bahrain \\ ${ }^{3}$ Department of Cardiology, King Hamad University Hospital, Kingdom of Bahrain
}

*Corresponding author: Maysa Salman, 22 Malone Beeches, BT9 6UB, Belfast, United Kingdom, Tel: +0044 $7498900777 / 00973$ 32002990; E-mail: maysah.almulla@gmail.com

Rec date: February 01, 2018; Acc date: February 13, 2018; Pub date: February 16, 2018

Copyright: $\odot 2018$ Salman M, et al. This is an open-access article distributed under the terms of the creative commons attribution license, which permits unrestricted use, distribution, and reproduction in any medium, provided the original author and source are credited.

\begin{abstract}
Introduction: Cardiovascular morbidity and mortality has been a tremendous cause of burden on the healthcare economy and is a major event in the Bahraini population. The same applies to vitamin D deficiency, which is a widely prevalent health problem, and in Bahrain more specifically. A limited amount of data has been published looking at the prevalence of vitamin D deficiency in patients with myocardial infarction, but only one study was done in the middle-eastern region.
\end{abstract}

Method: 50 patients with myocardial infarction (both ST elevation and non-ST elevation) were included in a 2month duration between May and July 2017. Inclusion criteria for the study were, age $\geq 30$ years, biomarker evidence of myocardial injury (elevated troponins or CKMB), other supporting evidence of MI (ECG charges, chest pain, hemodynamic instability). Low levels of vitamin D were considered as $<20 \mathrm{ng} / \mathrm{ml}$.

Results: Of the 50 enrolled patents (including both ST elevation and non-ST elevation MI), 43 (86\%) were found to have low vitamin D levels.

Conclusion: Vitamin D deficiency is present in most of the patients with acute myocardial infarction.

Keywords: Biomarkers; Myocardial infarction; Prevalence; Troponin; Vitamin D

\section{Introduction}

Cardiovascular morbidity and mortality has been a tremendous cause of burden on the healthcare economy and is a major event in the Bahraini population. The same applies to vitamin D deficiency, which is a widely prevalent health problem, and in Bahrain more specifically. Many factors contribute to low vitamin D status, including hospitalization, people with darker skin, medications that accelerate the metabolism of vitamin D as well as malabsorption conditions [1]. The main form of vitamin $\mathrm{D}$ that circulates in the body is 25hydroxyvitamin D, low levels are defined as $<20 \mathrm{ng} / \mathrm{ml}$. Vitamin D has been well-known as an important cause of musculoskeletal mortality, and there has been growing evidence that demonstrated the skeletal and extra-skeletal effects of vitamin D [2]. The cardiovascular system on the other hand seems to be a major target for the action of vitamin $\mathrm{D}$, however the mechanism is not fully understood to support this. The vitamin $\mathrm{D}$ receptors are present in both vascular smooth muscle and cardiomyocytes. Animal studies that looked at the relationship between vitamin $\mathrm{D}$ and cardiovascular incidents have suggested that by binding vitamin $\mathrm{D}$ to these receptors, a range of potentially beneficial cardiovascular benefits occur such as the relaxation of the smooth muscle and the reduction of atherosclerotic forming foam cells $[3,4]$. That forms the basis of the suggested relationship between vitamin $\mathrm{D}$ deficiency and myocardial infarction. A limited amount of data has been published looking at the prevalence of vitamin D deficiency in patients with myocardial infarction, but only one study was done in the middle-eastern region, KSA to be more exact. In this study, we demonstrated the prevalence of vitamin $\mathrm{D}$ deficiency in patients with myocardial infarction [5].

\section{Methods}

Recruitment of patients occurred from both the emergency department admission and patients developing MI in the inpatient wards after admission. 50 patients with myocardial infarction (both ST elevation and non-ST elevation) were included in a 2-month duration between May and July 2017. Inclusion criteria for the study were, age $\geq$ 30 years, biomarker evidence of myocardial injury (elevated troponins or CKMB), other supporting evidence of MI (ECG charges, chest pain, hemodynamic instability). Those patients with other cardiovascular diseases such as previous MI or cardiac surgery within 3 months prior to presentation as well as kidney impairment were not included in the cohort. Any patients on vitamin D supplementation were excluded. Detailed medical history examination and laboratory assessment of vascular risk factors was done. Vitamin D levels were obtained on presentation. For patients who had vitamin D levels checked in the 3 months prior to presentation, no new levels were ordered. Vitamin D deficiency was defined as 25-deoxyhydroxyvitamin D levels $\leq 20 \mathrm{ng} / \mathrm{ml}$ following the US preventive task force guidelines. Myocardial infarction was defined using the clinical symptoms, ECG abnormalities and blood biomarkers, following the American heart association 
Page 2 of 2

guidelines. Patients data included age, gender and ethnicity. Clinical variables included Hypertension, DM, Hyperlipidemia, CKD, previous history of heart diseases.

\section{Results}

During the period between May and July 2017, 50 patients were admitted under the cardiology department with myocardial infarction. The mean age was 57 amongst the cohort, $86 \%$ were males and $14 \%$ were females. The majority of patients (54\%) were Bahraini's. Of the 50 enrolled patents (including both ST elevation and non-ST elevation MI), 43 (86\%) were found to have low vitamin D levels (Figures 1-3).

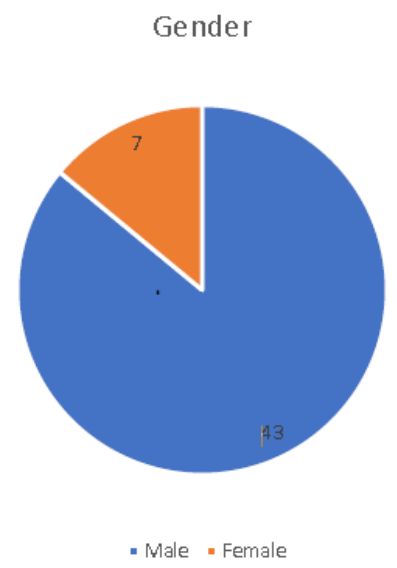

Figure 1: Gender.

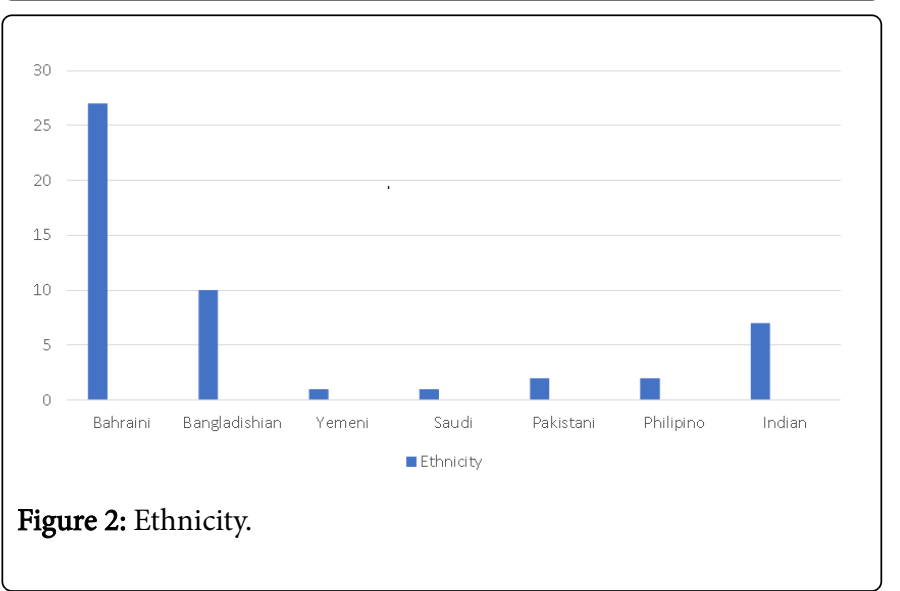

\section{Discussion}

This study demonstrated the prevalence of vitamin D deficiency in patients with myocardial infarction. The 3 main clinical variables that were highly associated with vitamin D deficiency were Hypertension, Diabetes and Hyperlipidemia respectively. Some animal studies suggested that vitamin D regulates RAS by suppressing renin gene expression, thus low levels of vitamin $\mathrm{D}$ leads to increased renin production and hypertension. There has been increasing evidence that vitamin $\mathrm{D}$ deficiency could be a causative factor in the development of both type 1 and type 2 diabetes. The mechanism suggested involves a reduction in insulin secretion secondary to vitamin $\mathrm{D}$ deficiency. Supplementation with vitamin $\mathrm{D}$ has been shown to restore insulin secretion in animals [6].

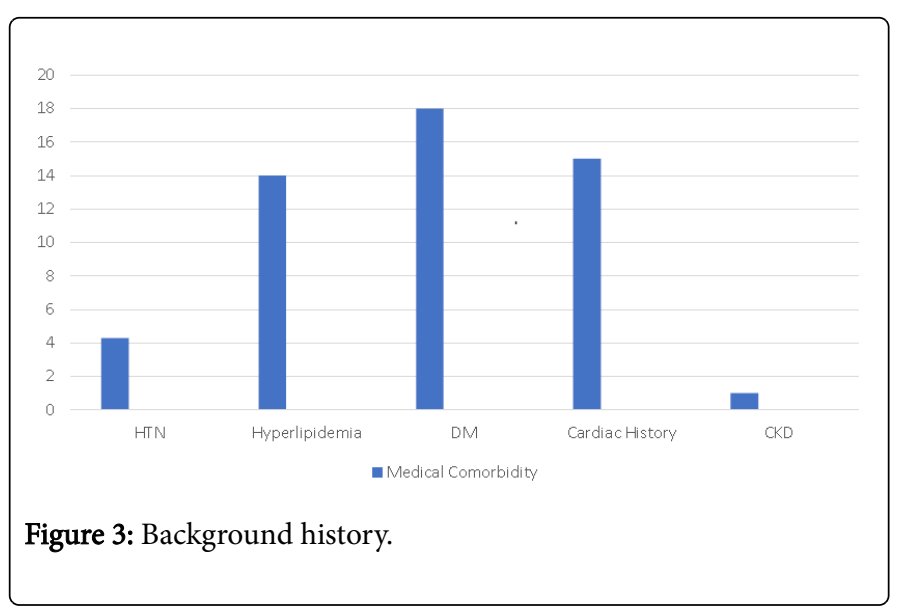

The cardiovascular system seems to be a major target for the action of vitamin D, its receptors are present in both vascular smooth muscle and cardiomyocytes. Animal studies have suggested that by binding vitamin $\mathrm{D}$ to these receptors, a range of potentially beneficial cardiovascular benefits occur such as the relaxation of the smooth muscle and the reduction of atherosclerotic forming foam cells. All above factors confirm an undeniable association between low levels of vitamin $\mathrm{D}$ and cardiovascular risks, including the development of myocardial infarctions. One huge limitation of this study was the small population size; thus, the results can't be a representative of the entire population and should not be generalized.

\section{Conclusion}

Vitamin D deficiency is evident in most of the patients with acute myocardial infarction Further studies are needed to investigate the effect of vitamin D supplementation in reducing the risk of cardiovascular diseases.

\section{References}

1. Muscogiuri G, Annweiler C, Duval G, Karras S, Tirabassi G, et al. (2017) Vitamin D and cardiovascular disease: From atherosclerosis to myocardial infarction and stroke. Int J Cardiol 230: 577-584.

2. Ng LL, Sandhu JK, Squire IB, Davies JE, Jones DJ (2013) Vitamin D and prognosis in acute myocardial infarction. Int J Cardiol 168: 2341-2346.

3. Aleksova A, Beltrami AP, Belfiore R, Barbati G, Nucci MD, et al. (2016) $\mathrm{U}$-shaped relationship between vitamin $\mathrm{D}$ levels and long-term outcome in large cohort of survivors of acute myocardial infarction. Int J Cardiol 223: 962-966.

4. Wimalawansa SJ (2016) Vitamin D and cardiovascular diseases: Causality. J Steroid Biochem Mol Biol 175: 29-43.

5. Matter M, El-Sherbiny E, Elmougy A, Abass A, Aldossary S, et al. (2016) Myocardial function in Saudi adolescents with vitamin D deficiency: Tissue Doppler imaging study. J Saudi Heart Assoc 28: 22-30.

6. Bourlon PM, Billaudel B, Faure-Dussert A (1999) Influence of vitamin D3 deficiency and 1,25 dihydroxyvitamin D3 on de novo insulin biosynthesis in the islets of the rat endocrine pancreas. J Endocrinol 160: $87-95$. 